\title{
Episodic hemilaryngopharyngeal spasm (HELPS) syndrome: case report of a surgically treatable novel neuropathy
}

\author{
Christopher R. Honey, MD, DPhil, FRCSC,1 Peter Gooderham, MD, FRCSC,1 \\ Murray Morrison, MD, FRCSC, ${ }^{2}$ and Zurab Ivanishvili, MD, FRCSC ${ }^{1}$ \\ Divisions of ${ }^{1}$ Neurosurgery and ${ }^{2}$ Otolaryngology, University of British Columbia, Vancouver, British Columbia, Canada
}

\begin{abstract}
The authors describe a novel cranial neuropathy manifesting with life-threatening episodic hemilaryngopharyngeal spasm (HELPS). A 50-year-old woman presented with a 4-year history of intermittent throat contractions, escalating to life-threatening respiratory distress. Botulinum toxin injections into her right vocal cord reduced the severity of her spasms, but the episodes continued to occur. MRI demonstrated a possible neurovascular conflict involving the cranial nerve IX-X complex and the posterior inferior cerebellar artery. Microvascular decompression of the upper rootlets of the vagal nerve eliminated her HELPS without complication. The authors propose a mechanism of HELPS implicating isolated involvement of the upper motor rootlets of the vagus nerve.
\end{abstract}

https://thejns.org/doi/abs/10.3171/2016.5.JNS16308

KEY WORDS episodic laryngospasm; hemilaryngopharyngeal spasm; vocal cord dysfunction; neurovascular conflict; microvascular decompression; HELPS syndrome; functional neurosurgery

$\mathrm{H}$ EMILARYNGOPHARYNGEAL spasm (HELPS) attributable to a neurovascular conflict in the cerebellopontine angle has not been previously reported. Well-recognized syndromes associated with vascular compression of the cranial nerves include trigeminal neuralgia (TN), hemifacial spasm (HFS), and glossopharyngeal neuralgia $(\mathrm{GFN})$. In addition, the term vago-glossopharyngeal neuralgia has been proposed for the rare condition in which GFN is associated with symptoms related to cranial nerve (CN) X. ${ }^{2,11}$ We report a case of episodic hemilaryngopharyngeal spasm (HELPS) without pain that was suggestive of isolated vagal nerve (special visceral efferent) involvement. We demonstrate a neurovascular conflict on the preoperative MRI and describe the intraoperative findings documenting the conflict. We report the long-term benefit following microvascular decompression (MVD) and propose a mechanism to explain the clinical manifestations. Our aim is to describe this condition of cranial nerve pathology and highlight its surgical cure.

\section{Case Report}

A 50-year-old woman presented to our neurosurgery service with a 4-year history of episodic "throat contractions" associated with an accompanying very distressing feeling of "choking" and a sensation of "tongue swelling." She had no associated pain in her face, ears, or throat, and there were no symptoms suggestive of insular epilepsy (aura or partial seizure). In between these episodes, she felt entirely normal with no difficulties swallowing or talking.

In hindsight, a decade before the throat contractions began, the patient reported an episodic sensation of tongue swelling. This was initially triggered by harsh fumes (e.g., paint). She was diagnosed with a latex allergy and prescribed antihistamines as needed for this symptom but felt it never helped. The sensation of tongue swelling continued intermittently and then began to be associated with a feeling of choking. During the last 4 years, this choking sensation had usually been triggered by prolonged or loud talking. It would last a few minutes and then spontaneously abate. The frequency, length, and severity of attacks slowly increased, and they began to occur spontaneously even while sleeping.

As a result, the patient presented to the emergency department on multiple occasions. Treatments with antihista-

ABBREVIATIONS $\mathrm{CN}$ = cranial nerve; GFN = glossopharyngeal neuralgia; HELPS = hemilaryngopharyngeal spasm; HFS = hemifacial spasm; MVD = microvascular decompression; PICA = posterior inferior cerebellar artery.

SUBMITTED February 5, 2016. ACCEPTED May 13, 2016.

INCLUDE WHEN CITING Published online July 8, 2016; DOI: 10.3171/2016.5.JNS16308. 
mines, epinephrine, and bronchodilators were ineffective, and typically the episodes stopped spontaneously. Not surprisingly, there was a very high level of anxiety during the attacks. On several occasions, the patient was treated with anxiolytics (e.g., lorazepam), and this appeared to reduce the severity of the spasms. This prompted one physician to recommend a psychiatric consultation, but the patient declined.

On two occasions the patient was intubated for respiratory distress and stridor and required admission to the ICU. Notes from the first intubation recorded "no swelling of the tongue, epiglottis or para-glottic structures" despite her reported sensation of a swollen tongue. The otolaryngology service made a diagnosis of episodic vocal cord dysfunction, and botulinum toxin treatments were initiated. This involved injections of the toxin into the right vocal cord every 8 weeks (the right side was chosen by convention for a presumed bilateral problem). As a result of this treatment, the severity of the spasms was reduced, but they continued at the same frequency. The patient was otherwise healthy. There was no family history of trigeminal neuralgia, HFS, GFN, seizures, or dystonia, and a review of systems was unremarkable.

On examination, the patient had a breathy voice (the result of botulinum toxin injections). Her facial and tongue sensation were intact to touch and pin-prick, pharyngeal sensation was normal to touch, and her gag reflex was symmetrical. Her extraocular, face, jaw, tongue, and palatal movements were normal. There was no dystonic posturing in her face. Peripheral muscle power and tone were normal, and there was no dysmetria in her extremities. The rest of her neurological and general examination results were unremarkable. Unfortunately, we were unable to capture an episode with video-laryngoscopy because the spontaneous occurrences were relatively brief and she refused to allow us to induce them in a controlled setting.

MRI of the patient's brain demonstrated a possible neurovascular conflict on the right side involving the cranial nerve $(\mathrm{CN}) \mathrm{IX}-\mathrm{X}$ complex and posterior inferior cerebellar artery (PICA) (Fig. 1). An MVD was performed without complication. The tonsillomedullary segment of the PICA $^{9}$ was looped between the upper 2 rootlets of CN X with tenacious arachnoid adhesions between the artery and the 2 rootlets (Fig. 2). The arachnoid adhesions were sharply dissected free (Fig. 3), and the artery was mobilized medially so that it was no longer distorting the vagus nerve rootlets (Fig. 4). Postoperatively, she had one episode of spasm the day after surgery and then no further spasms. Her episodic sensation of a swollen tongue also no longer occurred even after exposure to paint. At 1 year, she remains free of her life-threatening laryngopharyngeal spasms.

\section{Discussion}

We report a rare case of severe episodic HELPS due to a neurovascular conflict involving the upper rootlets of the vagus nerve $(\mathrm{CN} \mathrm{X})$ and the tonsillomedullary segment of the posterior inferior cerebellar artery (PICA). The term "vago-glossopharyngeal neuralgia" has been advocated for cases in which both CN IX and CN X are involved..$^{2,11}$
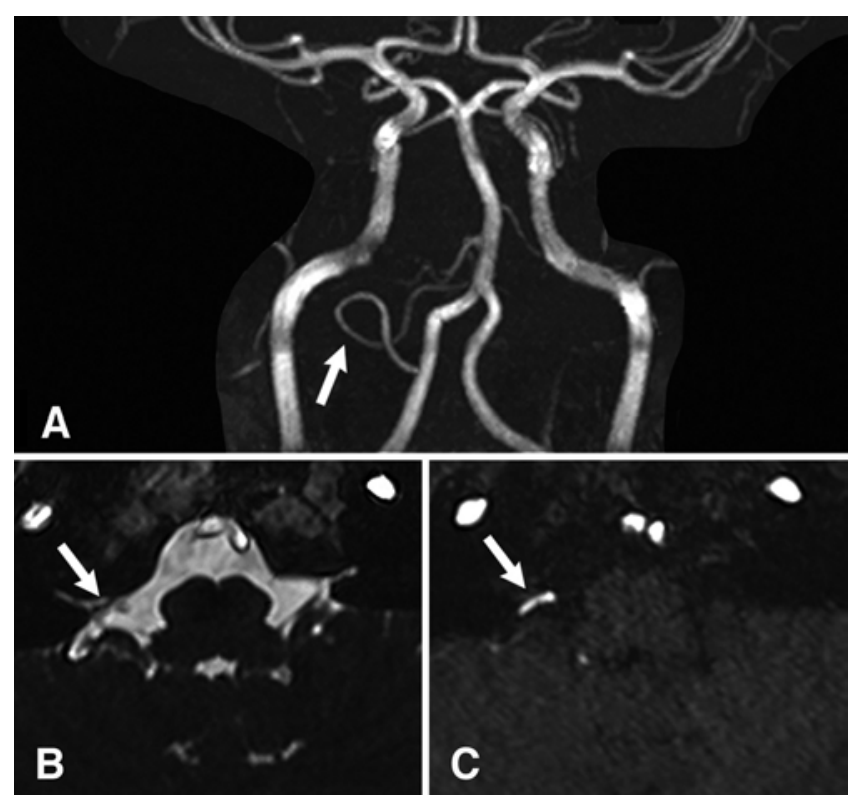

FIG. 1. Preoperative time-of-flight MR angiogram (A) demonstrating the prominent right PICA (arrow). Preoperative axial CISS (constructive interference in steady state) (B) and time-of-flight (C) images at the identical level. The arrows indicate the rostral entrance to the jugular foramen.

In these rare cases, the painful glossopharyngeal neuralgia syndrome is associated with cardiovascular events, such as severe bradycardia and asystole, or respiratory events, such as coughing paroxysms. ${ }^{1,4,5}$ One of the proposed mechanisms for the cardiac effects of vago-glossopharyngeal neuralgia includes a central reflex connection between the solitary nucleus, receiving afferents from $\mathrm{CN}$ IX, and the dorsal motor nucleus of the vagus, giving rise to the parasympathetic efferents in $\mathrm{CN} \mathrm{X}{ }^{8}$ Hyperactivity in the parasympathetic outflow can lead to bradycardia and asystole. Similarly, the central connections between

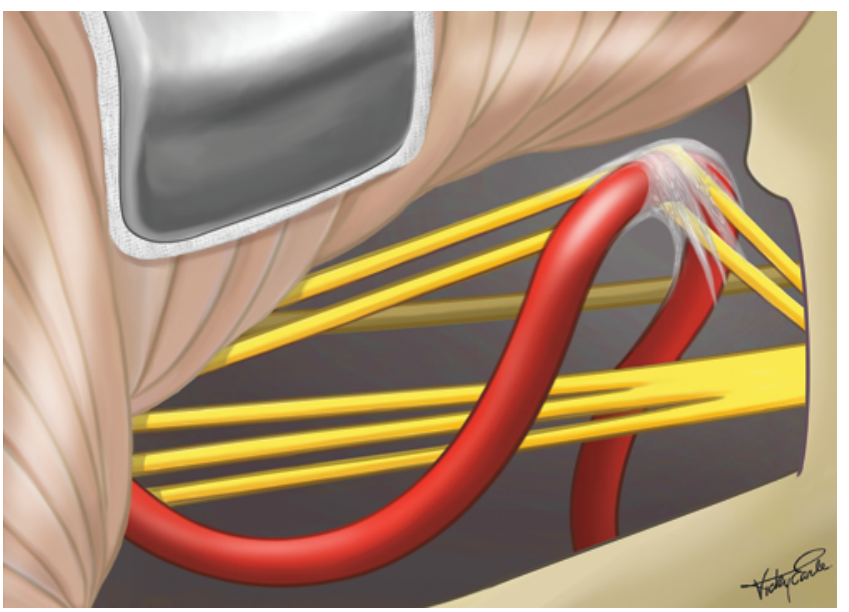

FIG. 2. Illustration of the operative view during microvascular decompression. The cerebellum has been retracted superomedially, revealing $\mathrm{CNs} I X$ and $\mathrm{X}$. Following removal of the overlying arachnoid curtain, the upper 2 rootlets of the vagus nerve are seen to be severely distorted by the tonsillomedullary segment of the PICA and caught by some arachnoid adhesions. Copyright Vicky Earle. Published with permission. 


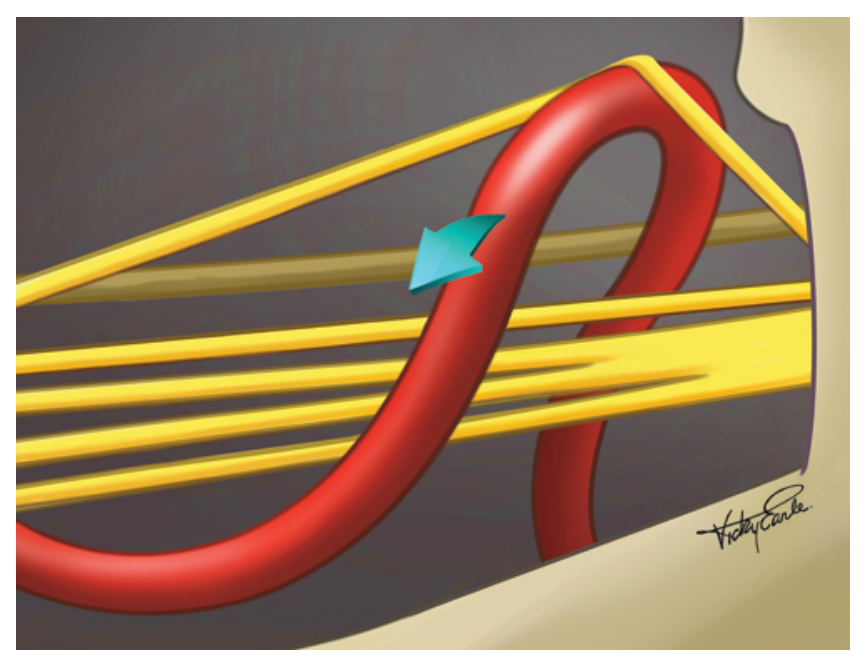

FIG. 3. Once the upper 2 nerve rootlets of the vagus nerve were sharply dissected free of arachnoid adhesions, one of them fell back into a normal position. The PICA was then mobilized medially to take the pressure off the upper most rootlet. Note that there was no compression of the glossopharyngeal nerve or the lower rootlets of the vagus nerve. Copyright Vicky Earle. Published with permission.

the solitary nucleus and the "cough network" in the brainstem $^{3}$ may explain the paroxysmal coughing episodes associated with the neuralgia pain in this condition.

Our patient did not report any paroxysmal pain, and she did not have symptoms associated with excessive parasympathetic vagal outflow. The clinical picture of our patient was more reminiscent of HFS - an intermittent, pure motor syndrome that progresses over time and can occur during sleep. The pathophysiology of HFS has been explained in terms of transaxonal short-circuiting between the branchial efferent fibers in CN VII as a result of pressure atrophy of the insulating myelin sheaths. ${ }^{7}$ Our patient exhibited symptoms that were associated with the visceral efferents of CN X, originating from the nucleus ambiguus. She exhibited paroxysmal laryngospasm compromising her airway. Botulinum toxin injections into her vocal cord on the right side were effective in reducing the severity of the spasms (similar to the standard treatment for HFS), but the treatment did not alleviate the episodes. MVD of CN $\mathrm{X}$ was curative in our case (again similar to HFS). This supports a peripheral compression of the motor rootlets of $\mathrm{CN} X$ as the putative cause of HELPS.

The reasons for the previous absence of reporting on HELPS may be due to a combination of its rare occurrence and underrecognition. This is the first case recognized at our center despite having examined more than 2000 patients with various neurovascular compression syndromes. The referral came to us from our otolaryngology team, and this condition (paroxysmal choking due to a neurovascular conflict) may just not yet be recognized by the otolaryngology community. Case reports of pharyngeal spasm in the context of multiple sclerosis have been published in which pharyngeal spasms are triggered by swallowing. ${ }^{10}$ The spasmodic conditions in these case reports have been successfully treated with carbamazepine. Our patient did not have multiple sclerosis, but we did not think to trial carbamazepine. If HELPS is indeed similar

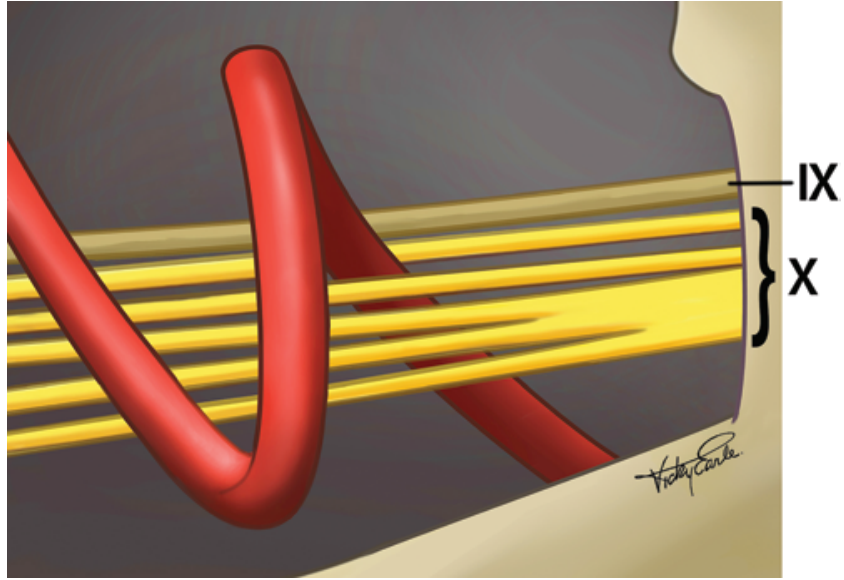

FIG. 4. Final position of the lower CNs and the tonsillomedullary segment of the PICA. Ultimately, the upper nerve rootlets of the vagus nerve were free from compression. Copyright Vicky Earle. Published with permission.

to HFS, then we would postulate that antiepileptic medications would not be effective. Paroxysmal choking has also been reported with insular epilepsy. These cases typically have the additional features of an aura (nausea or a rising epigastric sensation) and partial seizure.

Episodic laryngospasm has been reported in the otolaryngology literature and is felt to be due to a conversion disorder or occult gastroesophageal reflux. ${ }^{6}$ Symptoms in these reported cases of episodic paroxysmal laryngospasm have features similar to those in our case. For example, Gallivan et al. described sudden attacks of upper airway obstruction resulting in unnecessary endotracheal intubation. ${ }^{7}$ Laryngoscopy in those cases revealed bilateral laryngospasm. Our patient declined video-laryngoscopy during an induced attack, and we do not know if her vocal cords contracted symmetrically (perhaps reflexively) or not. Interestingly, one of her physicians did recommend a psychiatry consultation, but she declined.

We believe that the intermittent sensation of "a swollen tongue" may be part of the symptom complex because it invariably accompanied the later, more severe symptoms of laryngospasm and it completely resolved postoperatively. This symptom was not lateralized. The sensation may have been triggered by activation of the vagus innervated muscles of the soft palette (especially palatoglossus). We postulate that this syndrome could occur with either pure laryngospasm or laryngopharyngeal spasm, depending on which branches of the vagus were compressed. We have chosen to use the more encompassing term-hemilaryngopharyngeal spasm, or HELPS, syndrome-for this initial description.

\section{Conclusions}

To the best of our knowledge, we report the first case of episodic hemilaryngopharyngeal spasm that was cured with MVD. The episodic symptom complex consisted of 1) a sensation of a swollen tongue despite its normal appearance, 2) choking initially triggered by speaking then occurring spontaneously, and 3) laryngospasm prompting emergency consultations. Misdiagnosis as a psychiatric 


\section{R. Honey et al.}

or allergic condition will predictably lead to unsuccessful treatment. Conversely, overdiagnosis will lead to unnecessary neurosurgery, especially in light of the fact that vessels are often near the $\mathrm{CN}$ IX-X complex and imaging the rootlets of the vagus nerve can be difficult. With wider recognition of this syndrome, the patterns of muscle contraction (unilateral or bilateral laryngospasm, with or without pharyngeal involvement) will be better elucidated with video-laryngoscopy. The otolaryngology and neurosurgical communities should be aware of this rare but potentially life-threatening condition.

\section{Acknowledgments}

We thank Ms. Vicky Earle for the illustrations that accompany this paper.

\section{References}

1. Barbash GI, Keren G, Korczyn AD, Sharpless NS, Chayen M, Copperman Y, et al: Mechanisms of syncope in glossopharyngeal neuralgia. Electroencephalogr Clin Neurophysiol 63:231-235, 1986

2. Bruyn GW: Glossopharyngeal neuralgia. Cephalalgia 3:143-157, 1983

3. Canning BJ, Chang AB, Bolser DC, Smith JA, Mazzone SB, McGarvey L: Anatomy and neurophysiology of cough: CHEST Guideline and Expert Panel report. Chest 146:16331648,2014

4. Chen-Scarabelli C, Scarabelli TM: Neurocardiogenic syncope. BMJ 329:336-341, 2004

5. Esaki T, Osada H, Nakao Y, Yamamoto T, Maeda M, Miyazaki T, et al: Surgical management for glossopharyngeal neuralgia associated with cardiac syncope: two case reports. Br J Neurosurg 21:599-602, 2007
6. Gallivan GJ, Hoffman L, Gallivan KH: Episodic paroxysmal laryngospasm: voice and pulmonary function assessment and management. J Voice 10:93-105, 1996

7. Gardner WJ: Concerning the mechanism of trigeminal neuralgia and hemifacial spasm. J Neurosurg 19:947-958, 1962

8. Kandan SR, Khan S, Jeyaretna DS, Lhatoo S, Patel NK, Coakham HB: Neuralgia of the glossopharyngeal and vagal nerves: long-term outcome following surgical treatment and literature review. Br J Neurosurg 24:441-446, 2010

9. Lister JR, Rhoton AL Jr, Matsushima T, Peace DA: Microsurgical anatomy of the posterior inferior cerebellar artery. Neurosurgery 10:170-199, 1982

10. Restivo DA, Solaro C, Maimone D, Pavone A, MarcheseRagona R: Pharyngeal painful tonic spasms: paroxysmal painful swallowing. Ann Intern Med 155:649-650, 2011

11. White J, Sweet W: Idiopathic vagoglossopharyngeal neuralgia, in Pain and the Neurosurgeon: A Forty-Year Experience. Springfield, IL: Charles C Thomas, 1969, pp 265-302

\section{Disclosures}

The authors report no conflict of interest concerning the materials or methods used in this study or the findings specified in this paper.

\section{Author Contributions}

Conception and design: Honey, Morrison. Drafting the article: Honey. Critically revising the article: Gooderham, Morrison, Ivanishvili. Approved the final version of the manuscript on behalf of all authors: Honey.

\section{Correspondence}

Christopher R. Honey, Department of Neurosurgery, University of British Columbia, 2775 Laurel St., Ste. 8105, Vancouver, BC V5Z 1M9, Canada. email: chris.honey@telus.net. 